connectivity is proposed, rather than loss of neurons, to explain the neurological and cognitive deficits in preterm babies.

\title{
CSF OBSTRUCTION AND MALABSORPTION IN CONGENITAL HYDROCEPHALUS
}

The relative contribution of CSF malabsorption and obstruction in three different etiological groups of neonatal high-pressure hydrocephalus (HC) was assessed in a study at University of Bonn, Germany, and University of Groningen, The Netherlands. CSF biomarkers, transforming growth factor beta-1 (TGF B-1), and aminoterminal propeptide of type 1 collagen (PC1NP), indicative of growth factor- and fibrosis-related CSF malabsorption, were assessed and compared in neonates with post-hemorrhagic $\mathrm{HC}(\mathrm{n}=6)$, non-hemorrhagic triventricular HC $(n=4)$ and spina bifida (SB) HC $(n=12)$. CSF interleukin6 (IL-6) cytokines, indicative of inflammation, were low and did not differ between groups. TGF B-1 concentrations were significantly higher in post-hemorrhagic $\mathrm{HC}$ cases (median $355 \mathrm{pg} / \mathrm{ml}$ ) than in SB HC (median 103) and non-hemorrhagic HC (median 120); $\mathrm{p}=0.01$ and 0.03 , respectively. Median CSF PC1NP concentrations were significantly lower in SB HC $(180 \mathrm{ng} / \mathrm{ml})$ than in post-hemorrhagic $\mathrm{HC}(1,060 \mathrm{ng} / \mathrm{ml}) ; \mathrm{p}=0.002$. Neonatal posthemorrhagic $\mathrm{HC}$ is associated with high concentrations of CSF malabsorption-related biomarkers whereas SB and non-hemorrhagic HC have lower concentrations, indicating that CSF obstruction contributes more to the development of these cases than malabsorption. (Heep A, Bartmann P, Stoffel-Wagner B et al. Cerebrospinal fluid obstruction and malabsorption in human neonatal hydrocephaly. Childs Nerv Syst October 2006;22:1249-1255). (Respond: Dr Axel Heep, Department of Neonatology, University of Bonn, Adenauerallee 119, 53113 Bonn, Germany).

COMMENT. High TGF B-1 and PC1NP CSF concentrations in neonatal posthemorrhagic $\mathrm{HC}$ are indicative of a fibrosis-related malabsorption as the cause of the $\mathrm{HC}$, contrasting with relatively low levels of malabsorption biomarkers in SB and nonhemorrhagic triventricular HC. CSF obstruction, rather than malabsorption, plays a major role in the pathogenesis of high-pressure SB and non-hemorrhagic $\mathrm{HC}$.

\section{BRAIN TUMORS}

\section{BONE MINERAL DENSITY REDUCTION FOLLOWING IRRADIATION OF BRAIN TUMORS}

Total body bone mineral density (TBBMD) was measured by X-ray absorptiometry in 46 brain tumor patients aged from 3.8 to 28.7 years (mean $14.9 \mathrm{y}$ ) at a mean of $6.4 \mathrm{y}$ (range 1.4-14.8 y) after end of treatment for brain tumor. Tumors were astrocytic, grade 1, in 25 patients. Tumors were resected in 45 and biopsied in 1. Eleven received chemotherapy. Radiotherapy was cranial in 10 and craniospinal in 5. One third of the patients had low TBBMD ( $\mathrm{z}$ scores $<-2.0$ ). Only combined craniospinal irradiation was significantly associated with low z scores, while cranial irradiation showed a borderline statistical association. (Pietila S, Sievanen H, Ala-Houhala M, et al. Bone mineral density is reduced in 
brain tumour patients treated in childhood. Acta Paediatrica Oct 2006;95:1291-1297). (Respond: Dr S Pietila, Department of Paediatrics, Tampere University Hospital, PO Box 2000, FI-33521 Tampere, Finland).

COMMENT. The cause of the osteoporosis among children treated for brain tumor is multifactorial, and includes craniospinal irradiation. Other possible factors are glucocorticoid treatment, motor handicaps, and impaired nutrition. Early identification could lead to effective therapy.

\section{DEMYELINATING DISEASES}

\section{CLINICAL MANIFESTATIONS OF MULTIPLE SCLEROSIS IN TAIWANESE CHILDREN}

Twenty-one patients with multiple sclerosis (MS) and onset before 18 years were treated over the past 22 years and their records retrospectively analyzed at the National Taiwan University Hospital, Taipei, and Min-Sheng General Hospital, Taoyuan, Taiwan. Fifteen were female and 6 male, mean age $12.4+/-4.5$ years. Presenting symptoms or signs in order of frequency were limb weakness $(62 \%)$, visual loss or field defect $(43 \%)$, bulbar symptoms $(33 \%)$, sensory disturbance $(29 \%)$, headache $(29 \%)$, ataxia $(19 \%)$, bowel or sphincter dysfunction (14\%), and encephalopathy or encephalitis (14\%). Multiple symptoms occurred at onset in $76 \%$. A viral prodrome, usually upper respiratory, was reported 2 weeks before onset of MS symptoms in $43 \%$.

MRI at onset showed lesions in the cerebral white matter in $72 \%$, most commonly in periventricular white matter $(56 \%)$, in basal ganglia $(33 \%)$, cerebellum $(28 \%)$, spinal cord $(28 \%)$, corpus callosum $(22 \%)$, and optic nerve $(17 \%)$. Visual evoked potentials were abnormal in $77 \%$, and a total of $62 \%$ had optic nerve involvement. Only one had opticospinal MS. Of 9 patients receiving periodic subcutaneous interferon beta-1a, $4(44 \%)$ had no relapses. The course was relapsing remitting in $86 \%$, and secondary progressive in $14 \%$. The mean interval between the first and second attack was $7.2+/-10$ months, most occurring within 12 months. Three (14\%) patients who were initially diagnosed with acute disseminated encephalomyelitis developed MS after 4 months, 2 and 6 years intervals. (Weng W-C, Yang C-C, Yu T-W et al. Multiple sclerosis with childhood onset: Report of 21 cases in Taiwan. Pediatr Neurol November 2006;35:327-334). (Respond: Dr Wang-Tso Lee, Department of Pediatrics, National Taiwan University Hospital, 7 Chung-Shan South Road, Taipei, Taiwan).

COMMENT. Compared with the West, Asian populations appear to be less susceptible to MS, suggesting a genetic racial factor in susceptibility. The concordance rate for MS among monozygotic twins is $26 \%$ compared to $2 \%$ for dizygotic twins (Ebers GC et al. N Engl J Med 1986;315:1638). A 50-fold increase in risk for daughters is noted in female MS patients (Sadovnick AD et al. Neurology 1988;38:990-991). The female to male sex ratio has increased over the past 50 years and now exceeds 3.2:1 in Canada (Orton S-M et al and Canadian Collaborative Study Group. Lancet Neurol Nov 2006;5:932-936). An environmental factor is suspected. Similar changes in sex ratio are observed in USA, Australia, and Japan. In the above Taiwan series, the female to male ratio is 2.5:1. An MS 